
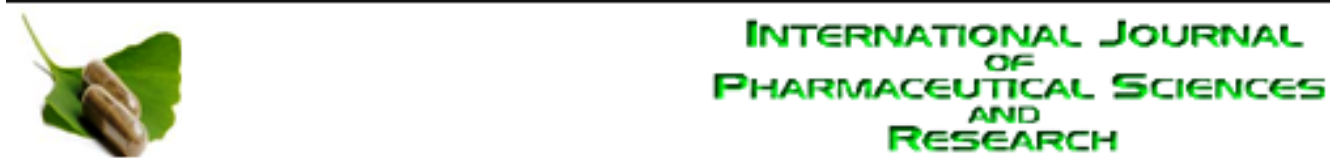

Received on 27 January, 2012; received in revised form 21 March, 2012; accepted 29 April, 2012

\title{
LIQUISOLID TECHNIQUE FOR ENHANCEMENT OF DISSOLUTION PROPERTIES OF FENOFIBRATE
}

\author{
P.M. Sabale* ${ }^{1}$, N.D. Grampurohit ${ }^{1}$, D.D.Gaikawad ${ }^{1}$, M.V.Gadhave ${ }^{1}$, G.M. Shingade ${ }^{2}$, G. Shaik ${ }^{2}$
}

Department of Pharmaceutics, Vishal Institute of Pharmaceutical Education \& Research ${ }^{1}$, Ale, Pune -412411 , Maharashtra, India

K.T. Patil College of Pharmacy ${ }^{2}$, Osmanabad- 413 501, Maharashtra, India

\begin{abstract}
Keywords:
Fenofibrate (FN),

Liquisolid System(LS),

Dissolution rate
\end{abstract}

Correspondence to Author:

Pramod Malhari Sabale

Department of Pharmaceutics, Vishal Institute of Pharmaceutical Education \& Research, Ale, Pune-412411,

Maharashtra, India

\section{ABSTRACT}

The in vitro dissolution property of slightly water soluble Fenofibrate (FN) was improved by exploring the potential of Liquisolid system (LS). The in vitro release pattern of LS compacts and directly compressed tablets were studied using USP-II apparatus. Different LS compacts were prepared using a mathematical model to calculate the required quantities of powder and liquid ingredients to produce acceptably flowable and compressible admixture. Avicel PH 102, Aerosil 200 and Explotab were employed as carrier, coating material and disintegrants respectively for preparing LS compacts. The prepared LS compacts were evaluated for their flow properties such as bulk density, tapped density, angle of repose, Carr's compressibility index and Hausner's ratio. The interaction between drug and excipients in prepared LS compacts were studied by differential scanning calorimetry (DSC) and X- ray powder diffraction (XRPD). The drug release rates of LS compacts were distinctly higher as compared to directly compressed tablets, which show significant benefit of LS in increasing wetting properties and surface area of drug available for dissolution. The LS1 of LS powder system showed acceptable flowability, Carr's compressibility index and Hausner's ratio. The DSC and XRD studies conforms the no significant interaction between the drug and excipients used in LS compacts. From this study it concludes that the LS technique is a promising alternative for improvement of dissolution property of water-insoluble drugs.

INTRODUCTION: In aqueous systems, Fenofibrate (FN) has a poor solubility and displays no amphiphilic character. Therefore the drug is molecularly dispersed in water until the solubility product of FN is exceeded. Schubert et al reported that, the solubilization of FN was linearly increased on addition of lecithin ${ }^{1}$. The poor dissolution rate of such water-insoluble drugs confronts a major obstacle in development of pharmaceutical dosage forms. The oral absorption of these drugs is often controlled by dissolution in $\mathrm{Gl}$ tract.
Thus dissolution of drug is of prime importance in absorption. The different techniques used to enhance the dissolution of water insoluble drugs, some of them are particle size reduction, surfactant as solublizing agent, drug complex with hydrophilic carrier, pro-drug approach, formulation of drug as solid solution to improve the dissolution rate by decreasing the crystallinity ${ }^{2}$. Among these the most promising method for promoting dissolution is the use of LS compacts $^{3-5}$. 
The term 'Liquisolid Systems' (LS) is a powdered form of liquid drug formulated by converting liquid lipophilic drug or drug suspension or solution of water-insoluble solid drug in suitable non-volatile solvent systems, into dry looking, non-adherent, free-flowing and readily compressible powdered mixtures by blending with selected carrier and coating materials. Various grades of cellulose, starch, lactose, etc. are used as the carriers, whereas very fine silica powder is used as the coating (or covering) material.

The good flow and compression properties of LS may be attributed due to large surface area of silica and fine particle size of avicel. Hence LS compacts containing water-insoluble drugs expected to display enhanced dissolution characteristics and consequently improved oral bioavailability.

In the present investigation, $\mathrm{FN}$ a very slightly water soluble drug was formulated into LS compacts consisting of similar powder excipients with different liquid vehicles. The in-vitro drug dissolution rates of such preparations were compared to those of conventionally prepared directly compressed tablets using a USP-II apparatus. DSC and XRPD technique were used to ascertain any interaction and crystallinity changes of drug in LS compacts due to interaction between drug and other excipients.

A Liquid Vehicle: Propylene glycol (PG), Polyethylene glycol 600 (PEG-600) ; b 8 mg of drug was incorporated in each tablet from liquid medication. ;c Each tablet contain $5 \% \mathrm{w} / \mathrm{w}$ of explotab.

\section{MATERIALS AND METHOD:}

Materials: Following gift samples were received: Fenofibrate (Alembic Research Ltd.,Vadodara-India.) Avicel PH 102 and Aerosil 200 Following samples were purchased:. S. D. Fine (Mumbai, India). Propylene glycols, PEG 600 Methanol, All reagents used were of analytical grade.

Solubility Studies: Solubility study of FN was carried out in Distilled water, Propylene glycol and PEG 400. Saturated solutions of FN were prepared in vehicles and kept in orbital shaker for $48 \mathrm{~h}$ at $25^{\circ} \mathrm{C}$. After this period, the solutions were filtered, diluted and analysed by UV-spectrophotometer (Jasco v430, Japan) at $245 \mathrm{~nm}$. Three determinations were carried out for each sample to calculate the solubility of FN. The results were extrapolated to determine the percent $\mathrm{w} / \mathrm{w}$ of FN in its saturated solution with the solvent under investigation.

Calculation of Liquid Load Factors ${ }^{6-7}$ : From reported $\Phi$-value the liquid load factor (Lf) and quantities of carrier and coating materials were calculated by using following formulae

$$
\phi L f=\Phi+\phi(1 / R)(1)
$$

$\Phi$ - Flowable retention potential for carrier material $\phi$ - Flowable retention potential for coating material

$$
\begin{array}{ll}
\text { - } & \mathrm{Lf}=\mathrm{W} / \mathrm{Q}(2) \\
\text { - } & \mathrm{Qo}=\mathrm{W} / \mathrm{Lo}(3) \\
\text { - } & \mathrm{qo}=\mathrm{Qo} / \mathrm{R}(4)
\end{array}
$$

Preparation of Conventional Tablet and Liquisolid compacts $^{8-10}$ : Conventional tablets of FN (DC-1) were prepared by direct compression using manual tablet machine, each containing $8 \mathrm{mg}$ drug with Avicel $\mathrm{PH}$ 102, lactose monohydrate, Aerosil 200 and explotab. Various LS compacts (LS-1 to LS-11) containing $8 \mathrm{mg}$ of FN were prepared by dispersing in nonvolatile vehicles such as propylene glycol and PEG 400 . Then a binary mixture of carrier (Avicel PH 102) and coating material (Aerosil- 200) was prepared at a ratio of 20:1. This binary mixture was added to the admixture of drug and vehicle.

Depending upon the type of vehicle in the formulation, different liquid load factors were employed in LS preparations. Therefore, different concentrations of Avicel and silica were used to prepare different Liquisolid formulations. Finally explotab as disintegrant was added in above powder blend and mixed. The final powder blend was subjected to compression. Important formulation characteristics of LS compacts are shown in Table 1. 
TABLE 1: KEY FORMULATION CHARACTERISTICS OF PREPARED FN LIQUISOLID COMPACTS

\begin{tabular}{|c|c|c|c|c|c|}
\hline Liquisolid system & $\begin{array}{c}\text { Non-volatile } \\
\text { solvent a }\end{array}$ & $\begin{array}{l}\text { Drug concentration in liquid } \\
\text { medication } \mathrm{Cd}(\% \mathrm{w} / \mathrm{w}) \mathrm{b}\end{array}$ & $\begin{array}{l}\text { Liquid load factor } \\
\text { (Lf) }\end{array}$ & $\begin{array}{c}\text { Tablet weight } \\
\text { (mg) c }\end{array}$ & $\begin{array}{c}\text { Molecular } \\
\text { fraction (FM) }\end{array}$ \\
\hline LS-1 & PG & 10 & 0.232 & 459.3 & 0.958 \\
\hline LS-2 & PG & 12.5 & 0.233 & 364.9 & 0.767 \\
\hline LS-3 & PG & 15 & 0.235 & 300.8 & 0.634 \\
\hline LS-4 & PG & 17.5 & 0.237 & 255.3 & 0.548 \\
\hline LS-5 & PG & 20 & 0.239 & 222.0 & 0.479 \\
\hline LS-6 & PG & 22.5 & 0.242 & 195.6 & 0.426 \\
\hline LS-7 & PG & 25 & 0.246 & 174.7 & 0.384 \\
\hline LS-8 & PG & 30 & 0.252 & 144.3 & 0.319 \\
\hline LS-9 & PG & 40 & 0.252 & 108.1 & 0.240 \\
\hline LS-10 & PG & 50 & 0.252 & 86.5 & 0.192 \\
\hline LS-11 & PEG & 10 & 0.202 & 514.2 & $0.616 \mathrm{~S}$ \\
\hline
\end{tabular}

Flow properties of Liquisolid system ${ }^{11}$ : Flow properties of the LS were estimated by tap density, bulk density, angle of repose, Carr's compressibility index and Hausner's ratio. Angle of repose was measured according to the fixed funnel method. The tap density was determined using bulk density apparatus and calculated the Carr's compressibility index and Hausner's ratio.

X-Ray Powder Diffraction: X-ray diffractograms of FN, excipients and LS formulations were performed by using Philips Analytical XRD (PW 3710). The cross section of samples was exposed to $\mathrm{X}$-ray radiation $(\mathrm{Cr})$ with scanning range from 10-70 02Ф.

Differential Scanning Calorimetry: Thermograms of FN, excipients and LS compacts were recorded on a DSC (Universal V2.4F TA instruments). Thermal behavior of the samples was investigated under a scanning rate of $10^{\circ} \mathrm{C} / \mathrm{min}$, covering a temperature range of $0-300^{\circ} \mathrm{C}$.

In vitro Dissolution Studies ${ }^{12-14}$ : The in-vitro release profiles of FN from DC, LS compacts were obtained using a dissolution test apparatus USP-II (Electro Lab). The dissolution study was carried out in $900 \mathrm{ml}$ of $0.1 \mathrm{~N}$ $\mathrm{HCl}$ as dissolution medium at $37^{\circ} \mathrm{C} \pm 2^{\circ} \mathrm{C}$ at $50 \mathrm{rpm}$. The samples ( $5 \mathrm{ml}$ )were withdrawn at 2 min intervals up to $30 \mathrm{~min}$ and $15 \mathrm{~min}$ interval from 30 to $60 \mathrm{~min}$. The dissolution medium was then replaced by $5 \mathrm{ml}$ of fresh dissolution fluid to maintain sink condition. The withdrawn samples were filtered and analyzed spectrophotometrically (Jasco v430, Japan) at 245 $\mathrm{nm}$. The mean of three determinations was used to calculate the drug release from each of the formulations.
RESULT AND DISCUSSION: FN was selected as the model drug for these studies since it is slightly water soluble drug and ideal candidate for testing the potential of rapid release LS compacts. The spectroscopy study of FN solutions obeyed Beer's law which was linear at concentration range from 5 to 60 $\mu \mathrm{g} / \mathrm{ml}$. The results of solubility study of FN are given in Table 2 and which shows higher solubility in propylene glycol as compared to others. In order to calculate the required ingredient quantities, the flowable liquidretention potentials ( $\Phi$ - values) of powder excipients were utilized.

\section{TABLE 2: SOLUBILITY OF FN IN VARIOUS SOLVENTS}

\begin{tabular}{cc}
\hline Solvent & Solubility (\%w/w) \\
\hline Distilled water & 0.0195 \\
Propylene glycol & 9.5693 \\
Polyethylene glycol-600 & 6.1496 \\
\hline
\end{tabular}

According to Louis et al., in propylene glycol, the $\Phi$ value was 0.16 for Avicel PH 102 and 1.5 for Aerosil 200. Liquid load factor was calculated from the flowable liquid-retention potential according to equation 1 using $R$ value as 20 . The appropriate quantities of carrier $(Q)$ were calculated using equation 2 . The optimum quantities of carrier (QO) and coating material (q0) were obtained from equation 3 and 4 respectively.

Flow properties of Liquisolid powder system are affected by physical, mechanical as well as environmental factors. Therefore different flow parameters were employed such as, the angle of repose $(\Phi)$, Carr's compressibility index and Hausner's ratio 13 and their results are presented in table $\mathbf{3}$. The LS-1 Liquisolid powder system showed acceptable flowability, Carr's index and Hausner's ratio 
Polymorphic changes of drug are important factor which might affect the dissolution rate and in turn bioavailability. So that it is necessary to study polymorphic changes of FN in LS compacts.

TABLE 3. FLOW PROPERTIES OF LIQUISOLID SYSTEM

\begin{tabular}{cccc}
\hline Liquisolid system & $\begin{array}{c}\text { Angle of repose } \\
(\boldsymbol{\theta}) \pm \text { S.D.* }\end{array}$ & $\begin{array}{c}\text { Carrs compressibility Index } \\
(\%) \pm \text { S.D.* }\end{array}$ & $\begin{array}{c}\text { Hausner's ratio } \\
\pm \text { S.D.* }\end{array}$ \\
\hline LS-1 & $32.45 \pm 0.56$ & $18.21 \pm 1.03$ & $1.02 \pm 0.01$ \\
LS-2 & $35.19 \pm 0.16$ & $20.16 \pm 0.93$ & $1.07 \pm 0.01$ \\
LS-3 & $36.69 \pm 0.09$ & $23.54 \pm 0.11$ & $1.13 \pm 0.02$ \\
LS-4 & $34.52 \pm 1.21$ & $26.27 \pm 0.23$ & $1.17 \pm 0.01$ \\
LS-5 & $35.64 \pm 0.44$ & $29.83 \pm 0.77$ & $1.13 \pm 0.02$ \\
LS-6 & $39.21 \pm 0.37$ & $32.41 \pm 0.74$ & $1.12 \pm 0.01$ \\
LS-7 & $37.29 \pm 0.01$ & $30.88 \pm 0.62$ & $1.14 \pm 0.03$ \\
LS-8 & $38.10 \pm 0.11$ & $29.34 \pm 0.34$ & $1.13 \pm 0.01$ \\
LS-9 & $37.46 \pm 1.12$ & $28.96 \pm 0.29$ & $1.12 \pm 0.01$ \\
LS-10 & $39.37 \pm 0.88$ & $31.13 \pm 1.18$ & $1.10 \pm 0.02$ \\
LS-11 & $34.13 \pm 0.31$ & $28.11 \pm 0.49$ & $1.03 \pm 0.01$ \\
\hline
\end{tabular}

*S.D. Standard deviation from mean

Figure 1 shows PXRD of pure FN, pure excipients, physical mixture and LS system. FN shows sharp peaks at $10.56,27.18,32.21,34.82$ and 54.41 at $2 \Phi$. LS powder showed sharp diffraction peak at 22.5 at $2 \Phi$ which shows only Avicel PH 102 maintained its crystalline state. Absence of characteristic peaks of FN in LS indicates $\mathrm{FN}$ is entirely converted in to amorphous or solubilized from. FN in LS can be a result of solubilization in liquid vehicle that may be absorbed and adsorbed on Avicel PH 102 and Aerosil 200. From this we can say that FN formed solid solution within the carrier matrix. The amorphization or solublization of FN may contribute in enhancement of dissolution rate.

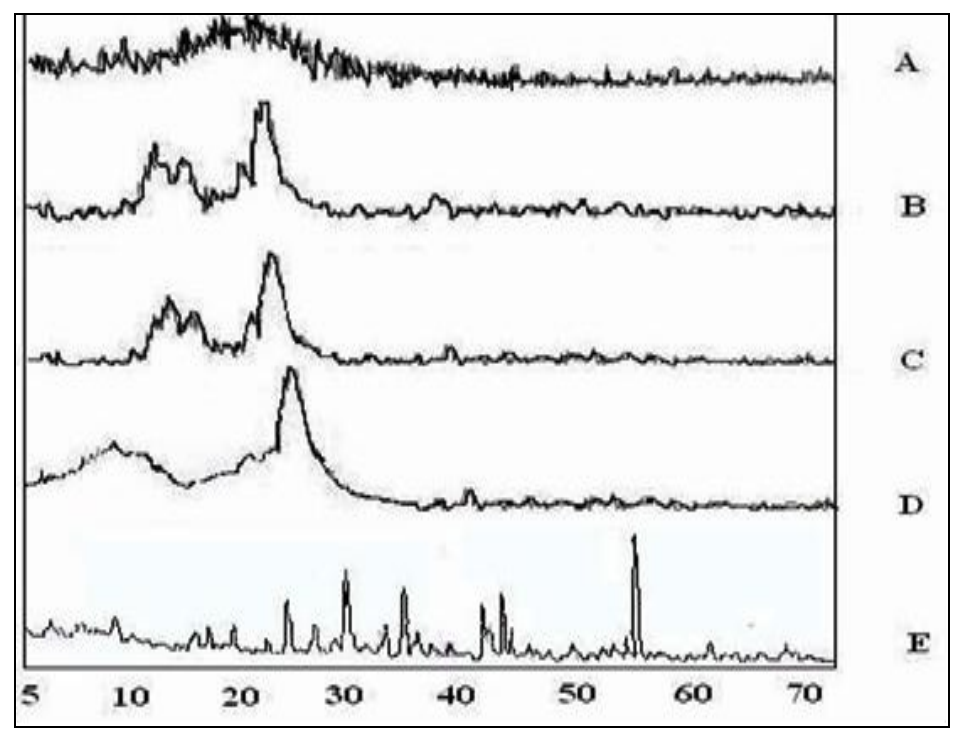

FIGURE 1: X-RAY DIFFRACTOGRAMS OF (A) AEROSIL 200, (B) AVICEL PH 102 (C) PHYSICAL MIXTURE (D) LIQUISOLID POWDER SYSTEM (E) PURE FN
To determine the possible interactions between a drug and excipients in LS compacts DSC was used. Figure $\mathbf{2}$ reveals the thermal behaviors of FN, excipients and LS. FN peaks are clear in its thermogram demonstrating a sharp characteristic endothermic peak at $234.76^{\circ} \mathrm{C}$ corresponding to its melting temperature; such sharp endothermic peak signifies that FN used was in pure crystalline state. The thermograms of Avicel PH 102 displayed broad endothermic peak at $106.18^{\circ} \mathrm{C}$ due to volatilization of adsorbed water and charring of the cellulosic material. The thermal behavior of Aerosil 200 did not show any sharp peaks; proving to be in an amorphous state.

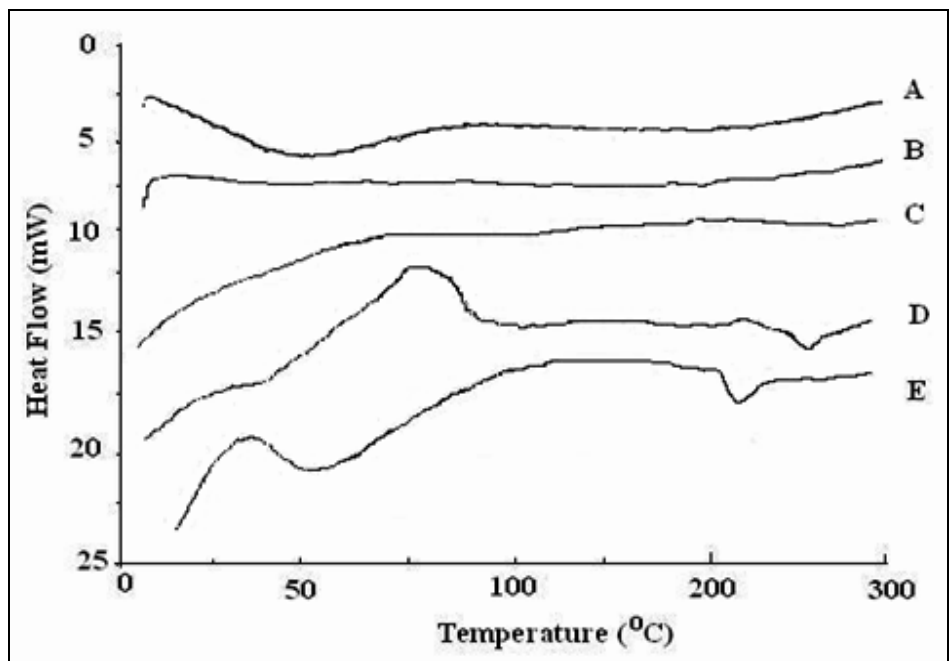

FIGURE 2: DSC THERMOGRAMS OF (A) AVICEL PH 102, (B) AEROSIL 200, (C) PHYSICAL MIXTURE,(D) LIQUISOLID POWDER SYSTEM, (E) PURE FN

On the other hand, the LS thermogram displayed complete disappearance characteristic peaks of FN; so we can say that formation of drug solution which is 
molecularly dispersed within the LS matrix. That was accompanied by the formation of a new endothermic peak at $265.27^{\circ} \mathrm{C}$ that indicates to the melting and decomposition of the whole LS. Such disappearance of the drug peaks upon formulation of the LS was surely indicating the formation of an amorphous solid solution.

Figure 3 shows the dissolution profile from the LS compact LS-1, LS-11 and directly compressed (DC-1) tablets of FN. Liquisolid compacts displayed distinct invitro release characteristics than directly compressed counterparts. The percent drug release at the end of $60^{\text {th }}$ min was, $95.89 \%$ for LS-1, $90.66 \%$ for LS-11 and $40.22 \%$ for DC-1.

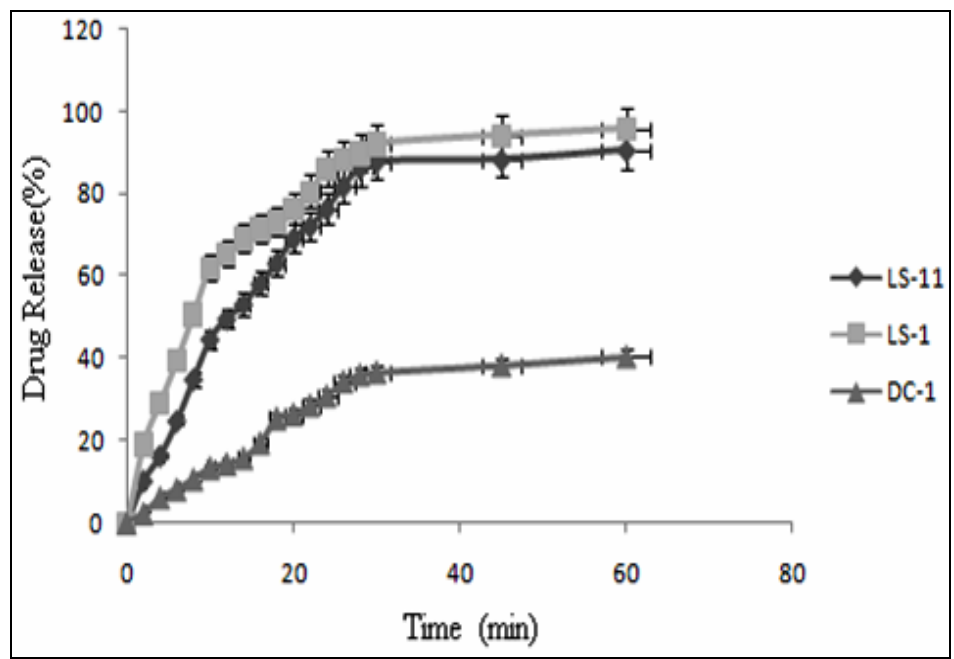

FIGURE 3: RELEASE PROFILE OF FN LIQUISOLID COMPACTS IN $0.1 \mathrm{NHCl}$ (Mean of three readings)

The $10^{\text {th }}$ min percent drug release of LS compacts and conventional tablets is shown in Figure 4. It was confirms that LS-1 had highest drug release $62.23 \%$ compared to $13.37 \%$ for conventional tablets (DC-1). Since the Liquisolid compacts contain a solution of the drug in Propylene glycol and PEG 400, the drug surface available for dissolution is tremendously increased. In essence, after disintegration, the LS primary particles suspended in the dissolving medium contain the drug in a state of molecular dispersion, whereas the directly compressed tablets are merely exposing micronized drug particles. Therefore, in the case of LS compacts, the surface area of drug available for dissolution is related to its specific molecular surface which, by any means, is much greater than that of the FN particles delivered by the directly compressed tablets. According to the Noyes and Whitney ${ }^{14} D \mathrm{R}=(D / h) S(C s-$ C) ${ }^{8}$.

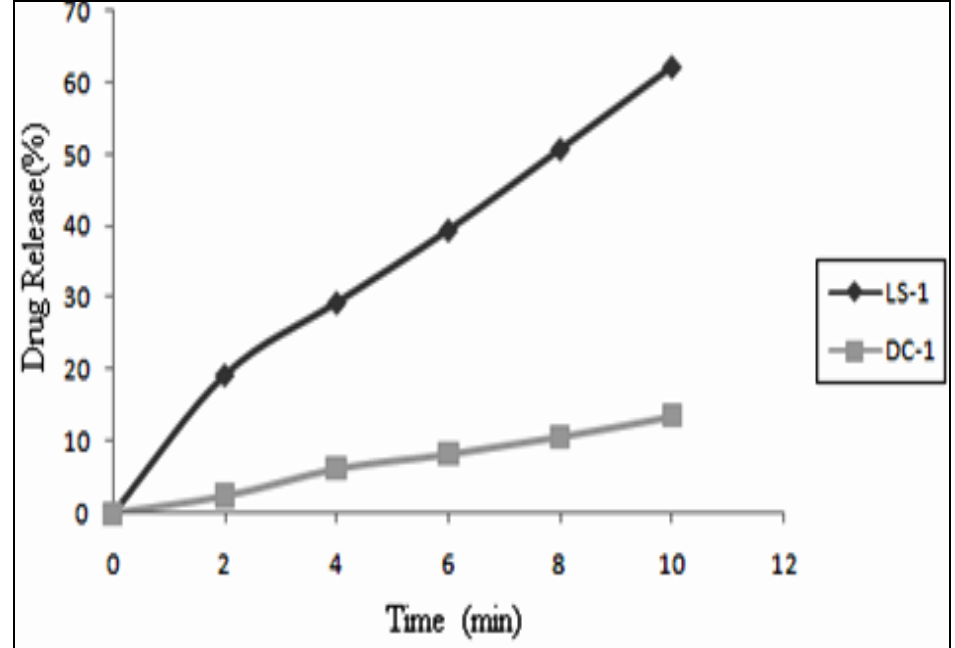

FIGURE 4: DRUG RELEASE (\%) AT 10 MIN FROM LIQUISOLID COMPACTS (LS-1) AND CONVENTIONAL TABLETS (DC-1)

The drug dissolution rate $(D R)$ is directly proportional notonly to the concentration gradient $(C s-C)$ of the drug in the stagnant diffusion layer, but also to its surface area $(S)$ available for dissolution. Moreover, since all dissolution tests for both FN preparations were carried out at a constant rotational paddle speed (50rpm) and identical dissolving media, it is assume that the thickness $(h)$ of the stagnant diffusion layer and the diffusion coefficient $(D)$ of the drug molecules transported through it remain almost identical under each dissolution condition. Therefore, the significantly increased surface area of the molecularly dispersed FN in the LS compacts may be principally responsible for their observed higher dissolution rates.

The consistent and higher dissolution rate displayed by LS compacts will improve the absorption of drug from GI tract. From a physicochemical point of view, it is well established that the inadequate dissolution of water insoluble drugs is the major reason for poor and erratic bioavailability, since it is the rate determining step in the absorption of water insoluble drugs. The liquid vehicle contained in the LS seems to have some effects on their drug dissolution properties, as shown in Figure 3. The relatively poorer dissolution properties of the LS-11 compact may be mainly attributed to the lower solubility of FN in PEG 600 compared to those in propylene glycol shown in Table 2.

In essence, the drug is in a partly dissolved state in the liquid medications of LS-11 compact. On the other hand, the drug is completely in solution in the LS-1 compact, thereby presenting improved dissolution properties. 
The drug concentration in liquid medication ( $\mathrm{Cd}$ ranging from 10 to $50 \% \mathrm{w} / \mathrm{w}$ ) has an apparent effect on $\mathrm{FN} 10^{\text {th }}$ min percent drug release shown by Liquisolid compacts as shown in figure 5. In LS system, with increase in drug concentration $(C d)$ there is decline in vitro drug release rates until it reaches minimum plateau with LS compacts containing more than $25 \% \mathrm{w} / \mathrm{w}$ of drug concentration. Apparently, the solubilization and molecular dispersion states of the drug in LS are different.

In other words, the ratio of the drug's saturation solubility $(C L)$ in the liquid vehicle over the drug concentration $(C d)$ in the liquid medication carried by each system denotes the fraction (FM) of the dissolved, or molecularly dispersed drug in the liquid medication of the prepared LS compacts.

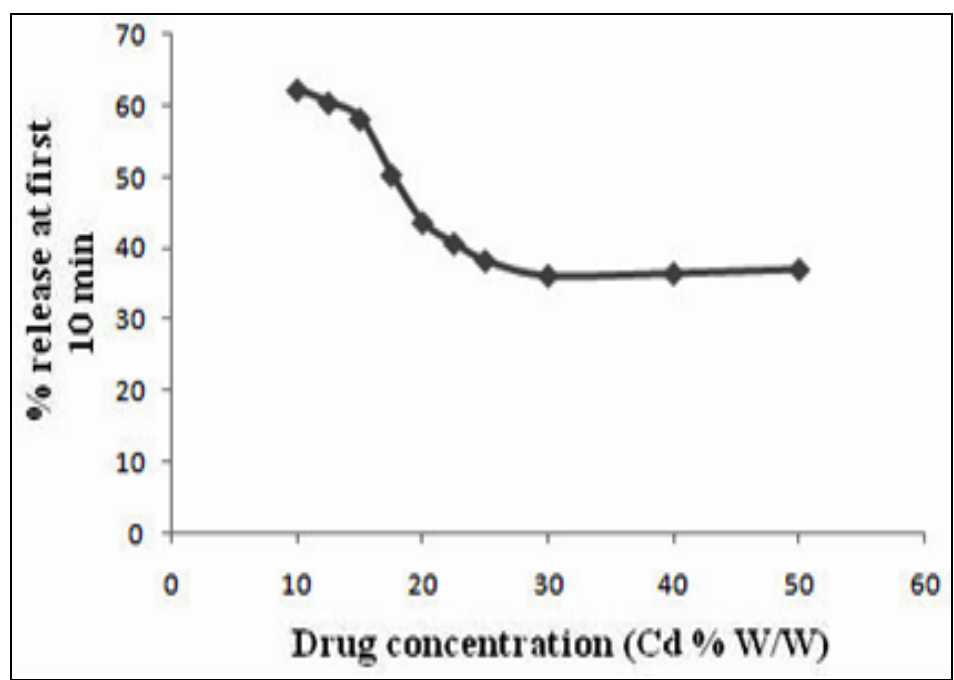

FIGURE 5: THE EFFECT OF THE DRUG CONCENTRATION IN LIQUID MEDICATION ON 10 MIN RELEASE RATES (\%) OF FN LIQUISOLID COMPACTS OF PROPYLENE GLYCOL

The FM values of each Liquisolid preparation have been calculated in Table 1. It should be noted that the fraction of molecularly dispersed drug in any system should not exceed unity. Moreover, since no liquid vehicle is involved in the case of directly compressed tablets which contain plain FN their FM value was taken as zero.

CONCLUSION: The Liquisolid technique is a promising alternative for improvement of dissolution property of water-insoluble drugs, such as FN.
The higher dissolution rate showed by Liquisolid compacts may imply enhanced oral bioavailability due to the increased wetting properties and surface of drug available for dissolution. The Liquisolid compact of FN made in propylene glycol showed better dissolution rate than FN with PEG 600 based upon solubility and molecular fraction $\left(F_{\mathrm{M}}\right)$ of the drug in their liquid medication.

ACKNOWLEDGEMENTS: Authors are thankful to Principal K.T. Patil College of pharmacy, Osmanabad.(M.S.) and also thankful to principal Vishal Institute of Pharma. Edu. \& Research, Ale (Pune) for allowing collaborative work and for providing the necessary facilities for experimental work.

\section{REFERENCES:}

1. Schubert MA and Müller-Goymann CC. Solubilization of Fenofibrate in aqueous lecithin dispersions: Physicochemical characterization of interactions between drug and carrier. Pharmazie. 2001; 56: 734-737.

2. Kapsi SG and Ayres JW. Processing factors in development of solid solution formulation of itraconazole for enhancement of drug dissolution and bioavailability. Int J Pharm. 2001; 229:193203.

3. Spireas SS and Sadu S. Enhancement of prednisolone dissolution properties using Liquisolid compacts. Int J Pharm. 1998; 166:177-188.

4. Khaled et. al. Formulation and evaluation of hydrochlorothiazide Liquisolid tablets. Saudi J Pharm. 2001; 222: 1-6.

5. Spireas et. al. Effect of powder substrate on the dissolution properties of methchrothiazide Liquisolid compacts. Drug Dev Ind Pharm. 1999; 25: 163-168.

6. Spireas SS. Theoretical and practical aspects of Liquisolid compacts, PhD Thesis, St. John's University, New York.1993.

7. Spireas et. al. Powdered solution technology: principles and mechanism. Pharm Res. 1992; 9:1351-1358.

8. Louis et. al. Improvement of dissolution properties of Carbamazepine through application of the Liquisolid tablet technique. Eur J Pharm Biopharm. 2008; 69: 342-347.

9. Spireas et al. In vitro release evaluation of hydrocortisone Liquisolid tablets. J Pharm Sci. 1998; 87: 867-872.

10. Spireas SS. United State Patent no 6423339, July 23. 2002

11. Staniforth J., Powder flow, in: M. Aulton (ed.), Pharmaceutics, the Science of Dosage Form Design,Churchill Livingstone, Longman group, Edinburgh, 2002. 2nd ed: pp. 197-210.

12. The United States Pharmacopoeia National Formulary; The United States Pharmacopoeial Convention, 2005:342-343.

13. Ansel et. al. Pharmaceutical Dosage Forms and Drug Delivery Systems, Lippincott Williams and Wilkins,Philadelphia. 1999; 7th ed: pp.60-63.

14. Noyes AA and Whitney WR. The rate of solution of solid substances in their own solutions. J Am Chem Soc.1897. 